\title{
The Effect of Rebamipide on Refractive Accuracy of Cataract Surgery in Patients with Dry Eye
}

\author{
Takeshi Teshigawara (D) · Akira Meguro (D) · Nobuhisa Mizuki (D)
}

Received: September 15, 2021 / Accepted: January 6, 2022 / Published online: January 22, 2022

(c) The Author(s) 2022

\begin{abstract}
Introduction: Refractive accuracy is crucial for increasing patient satisfaction after cataract surgery. Tear film instability caused by dry eye can lead to inaccurate measurement of corneal power, which is one of the most important factors for postoperative refractive calculation. This study investigated the effect of $2 \%$ rebamipide ophthalmic suspension on the predicted refractive accuracy for cataract surgery in patients with dry eye.

Methods: This single-center, prospective, openlabel study included 35 dry eyes (with tear breakup time $<5 \mathrm{~s}$ ) who underwent cataract surgery. The patients were treated with rebamipide for 4 weeks before the preoperative
\end{abstract}

Supplementary Information The online version contains supplementary material available at https:// doi.org/10.1007/s40123-022-00457-3.

T. Teshigawara $(\bowtie)$

Department of Ophthalmology, Yokosuka Chuoh Eye Clinic, 2-6 Odaki-cho, Yokosuka, Kanagawa 238-0008, Japan

e-mail: teshitake@gmail.com

T. Teshigawara

Tsurumi Chuoh Eye Clinic, 1-2-1 Tsurumi-chuo, Tsurumi, Yokohama, Kanagawa 230-0051, Japan

T. Teshigawara $\cdot$ A. Meguro $\cdot$ N. Mizuki Department of Ophthalmology, Yokohama City University School of Medicine, 3-9 Fukuura,

Kanazawa, Yokohama, Kanagawa 236-0004, Japan examination. In addition to the usual evaluation of biometric variables essential for cataract surgery, tear breakup time (TBUT), superficial punctate keratopathy in the central part of the cornea (C-SPK), corneal higher-order aberrations (HOAs), and predicted postoperative spherical equivalent (P-SE) were evaluated at baseline and 4 weeks after dry eye treatment. The patients' subjective spherical equivalent (SSE) was evaluated 1 month postoperatively. The difference between S-SE and P-SE was used to assess the accuracy of the P-SE. Improvement in TBUT, C-SPK, HOA, and the accuracy of P-SE after dry eye treatment were analyzed.

Results: The accuracy of the biometry readings for the achieved P-SE was within $0.25 \mathrm{D}$ in $42.9 \%$ and $54.3 \%$; within $0.5 \mathrm{D}$ in $71.4 \%$ and $88.6 \%$; and within $0.75 \mathrm{D}$ in $88.6 \%$ and $97.1 \%$ of eyes before and after rebamipide treatment, respectively $(p<0.01)$. TBUT, C-SPK, and HOAs significantly improved after rebamipide treatment $(p<0.01)$.

Conclusion: Rebamipide significantly improved the corneal surface condition and accuracy of the predicted postoperative refraction in dry eyes.

Keywords: Cataract surgery; Dry eye; Higherorder aberrations; Predicted postoperative refraction; Rebamipide; Superficial punctate keratopathy 


\section{Key Summary Points}

Why carry out this study?

Blurred vision has been ranked at the top of the list of postoperative patient dissatisfaction $(68 \%)$ in cataract surgery and is mainly (57\%) caused by the inaccuracy of predicted postoperative refraction.

One of the main causes of inaccuracy in predicted postoperative refraction is ocular surface irregularity, which is often induced by tear film instability in dry eyes.

We hypothesize that dry eye treatment with $2 \%$ rebamipide ophthalmic suspension may improve the accuracy of predicted postoperative refraction.

\section{What was learned from the study?}

This study demonstrated that preoperative dry eye treatment with rebamipide may be useful for improving the quality of ocular surface conditions, as evidenced by the improvement in tear breakup time, corneal higher-order aberrations, and superficial punctate keratopathy in the central part of the cornea.

Preoperative dry eye treatment with rebamipide can lead to an increase in the accuracy of predicting postoperative refraction.

Preoperative dry eye treatment with rebamipide may be an option for improving the accuracy of intraocular lens power calculation.

\section{INTRODUCTION}

Cataract is highly prevalent in Japan, with $24.2 \%$ of individuals in all age groups and $41.7 \%$ in those over 80 years of age requiring cataract surgery [1]. Currently, a high level of refractive accuracy is expected by patients undergoing cataract surgery [2]. Blurred vision has been ranked at the top of the list of postoperative patient dissatisfaction (68\%) and is mainly $(57 \%)$ caused by postoperative refractive error [3].

The accuracy of postoperative refraction relies on the accurate choice of intraocular lens (IOL) power. The IOL power calculation equations require various biometric factors, including corneal curvature, axial length (AL), and anterior chamber depth (ACD). Among these variables, corneal curvature is the most influential refractive factor in optical systems; therefore, accurate measurement is crucial for minimizing postoperative refractive error $[3,4]$. However, the accuracy of corneal power measurements has not improved significantly, despite marked improvements in AL and ACD measurements with the advent of optical coherence tomography and new biometrics approaches [5]. The inaccuracy of corneal power measurements is particularly significant in cases with ocular surface irregularity. The most common cause of preoperative ocular surface irregularity is dry eye [6].

Fluctuation and irregularity of the tear film can also cause intra-patient discrepancies in corneal power measurements [7]. Epitropoulos et al. [8] demonstrated a difference of as much as $0.5 \mathrm{D}$ in IOL power calculations during different visits in patients with dry eye.

Regarding the epidemiology of dry eye in patients undergoing cataract surgery, in the PHACO study [9], approximately $60 \%$ of patients showed rapid tear breakup time (TBUT), and about $35 \%$ of patients had superficial punctate keratopathy in the central part of the cornea (C-SPK). A previous study reported that higher-order aberrations (HOAs) measured in patients with dry eye and C-SPK were significantly higher than in patients with dry eye without C-SPK [10]. These results may imply that a significant number of patients undergoing cataract surgery may be vulnerable to ocular surface irregularity and inaccurate corneal power measurements.

Hovanesian et al. [11] reported that dry eye treatment with a 5\% lifitegrast ophthalmic 
solution improved corneal surface conditions, as evidenced by lowered corneal HOAs. They concluded that dry eye treatment with this solution improved the accuracy of the predicted postoperative refraction. However, 5\% lifitegrast was not available in the Japanese market until July 2021.

Rebamipide (Otsuka Pharmaceutical Co., Ltd., Tokyo, Japan) is a secretagogue that has the ability to increase mucin-like substances and decrease inflammatory cytokines, thereby increasing the stability of the tear film and decreasing ocular surface irregularity [12]. Moreover, the production of a mucin-like glycoprotein and expression of MUC1 and MUC4 were identified when human corneal epithelial cells were cultured with rebamipide [13], indicating that rebamipide may improve corneal epithelium damage, such as C-SPK [13]. However, no research has investigated whether dry eye treatment with rebamipide can improve the accuracy of predicted postoperative refraction, despite the clarification of the mechanism of action and effectiveness of rebamipide in dry eye.

Therefore, we hypothesized the following: First, ocular surface irregularity may be ameliorated by improving the condition of the corneal epithelium as well as tear film stability by the preoperative administration of rebamipide. Second, improved corneal surface conditions may increase the accuracy and decrease intrapatient discrepancies in corneal power measurement. Thus, postoperative refractive errors may be reduced.

Hence, in this study, we investigated the effect of dry eye treatment with rebamipide on the improvement of refractive accuracy in cataract surgery, with a view to improving the accuracy of IOL power calculation in dry eyes.

\section{METHODS}

The ethical committees of Yokosuka Chuo Eye Clinic and Tsurumi Chuo Eye Clinic approved this study. The study adhered to the tenets of the Declaration of Helsinki throughout the data collection process. After providing detailed information regarding this research, written informed consent was obtained from all patients for participation in this study and publication of the resulting data.

\section{Patients}

This single-center, prospective, open-label study included 35 eyes from 35 patients. All patients were scheduled for cataract surgery and were diagnosed with dry eye based on the Japanese dry eye diagnostic criteria of TBUT $\leq 5 \mathrm{~s}$ and the presence of symptoms such as pain, discomfort, or visual disturbance related to dry eye [14]. We assessed the subjective symptoms based on the Japanese version of the Ocular Surface Disease Index (J-OSDI) [15]. However, scoring the subjective symptoms is not included in the Japanese definition of dry eye; thus, we did not quantify those symptoms in this study.

Patients with the following medical history were excluded: ocular trauma, ocular inflammation, ocular scarring, dystrophy, and other conditions that can cause irregularity in the corneal surface. Additionally, patients with a history of previous ocular surgery or the use of contact lenses were excluded.

All patients received a 4 -week dry eye treatment with $2 \%$ rebamipide ophthalmic suspension before the preoperative evaluation of essential biometric variables for cataract surgery. All biometric variables were evaluated by the same examiner using the same device before and after dry eye treatment.

\section{Dry Eye Treatment}

Patients used only 2\% rebamipide ophthalmic suspension (Mucosta Ophthalmic Suspension UD2\%; Otsuka Pharmaceutical Co.) four times a day, for 4 weeks, before the preoperative examination for cataract surgery (use of any other eye drops was prohibited). Nurses checked the patients' treatment adherence on the examination date. 


\section{Examination of Tear Function, Ocular Surface Condition, Corneal Power, and Accuracy of Predicted Postoperative Spherical Equivalent}

Ocular evaluations were performed at baseline and after 4 weeks of dry eye treatment, as follows. The doctor used fluorescein dye to assess the ocular staining and TBUT. A fluorescein strip was moistened with saline, the excess fluid was removed, and the strip was placed close to the inferior bulbar conjunctiva. Patients were instructed to blink a few times. TBUT was then measured using a metronome, and the mean was calculated. Fluorescein corneal staining was evaluated in the center of the cornea per the National Eye Institute/Industry Workshop method [16]. The degree of C-SPK was scored from 0 to 3 , with $0=$ none, $1=$ mild, $2=$ moderate, and $3=$ severe. The CASIA 2 (Tomey Corporation, Nagoya, Japan) was used to assess HOAs within a $4-\mathrm{mm}$ area from the center of the cornea and also provided a detailed model of the optical properties of the cornea represented by Zernike polynomials. HOAs are a combined value of the magnitude of the third to sixth order calculated as the root mean square. Corneal power was measured using the IOLMaster 700 (Carl Zeiss Meditec AG, Jena, Germany). The predicted postoperative spherical equivalent (P-SE) was calculated with the Barrett Universal II IOL calculation formula [17].

Subjective postoperative visual acuity was evaluated 1 month postoperatively. The absolute difference between the subjective postoperative spherical equivalent (S-SE) and P-SE was regarded as the accuracy of P-SE. The accuracy of P-SE was compared between pre-and post-dry eye treatments. Additionally, the average accuracy of P-SE in both pre- and post-dry eye treatment calculated using the Barrett Universal II IOL calculation formula was compared with the accuracy of P-SE in a large-scale study in which dry eye was not considered as a criterion [18].

\section{Statistical Analysis}

All statistical analyses were performed using the Bell Curve for Excel software, 1.03 (Social Survey Research Information Co., Ltd., Tokyo, Japan). Post hoc power analysis was used to investigate the power of our tests for a given sample size $(n=35)$.

After dry eye treatment, the differences in C-SPK and HOAs and the accuracy of P-SE between pre- and post-dry eye treatment were analyzed using the Wilcoxon signed-rank test. A value of $p<0.05$ was considered statistically significant.

\section{RESULTS}

Thirty-five eyes of 35 patients (18 men and 17 women) were diagnosed with dry eye and were administered rebamipide for 4 weeks before preoperative examination for cataract surgery. The ages of the patients ranged from 62 to 85 years (mean, $74.5 \pm 6.2$ years).

The nurses confirmed that the treatment program was followed by all patients. There were no adverse effects during the investigation. The post hoc power analysis showed that for an effect size of $d=0.5$, Wilcoxon signed-rank tests had very good power (80.0\%).

Regarding essential biometric variables for the calculation of predicted postoperative refraction, the mean absolute differences (mean $\pm \mathrm{SD}$ ) between pre- and post-dry eye treatment were determined. The mean absolute differences in AL and ACD were $0.02 \pm 0.02 \mathrm{~mm}$ and $0.04 \pm 0.03 \mathrm{~mm}$, respectively. The mean absolute differences in steep and flat keratometry values were $0.27 \pm 0.14 \mathrm{D}$ and $0.29 \pm 0.16 \mathrm{D}$, respectively. The mean absolute difference in the meridian of the steep keratometric axis was $12.4^{\circ} \pm 9.4^{\circ}$.

The accuracy of P-SE was within $0.25 \mathrm{D}$ in $42.9 \%$ of eyes before and $54.3 \%$ of eyes after dry eye treatment, within $0.5 \mathrm{D}$ in $71.0 \%$ of eyes before and $88.6 \%$ of eyes after dry eye treatment, within $0.75 \mathrm{D}$ in $88.6 \%$ of eyes before and $97.1 \%$ of eyes after dry eye treatment, and within $1.0 \mathrm{D}$ in $97.1 \%$ of eyes before and $100 \%$ of eyes after dry eye treatment (Fig. 1). In each 


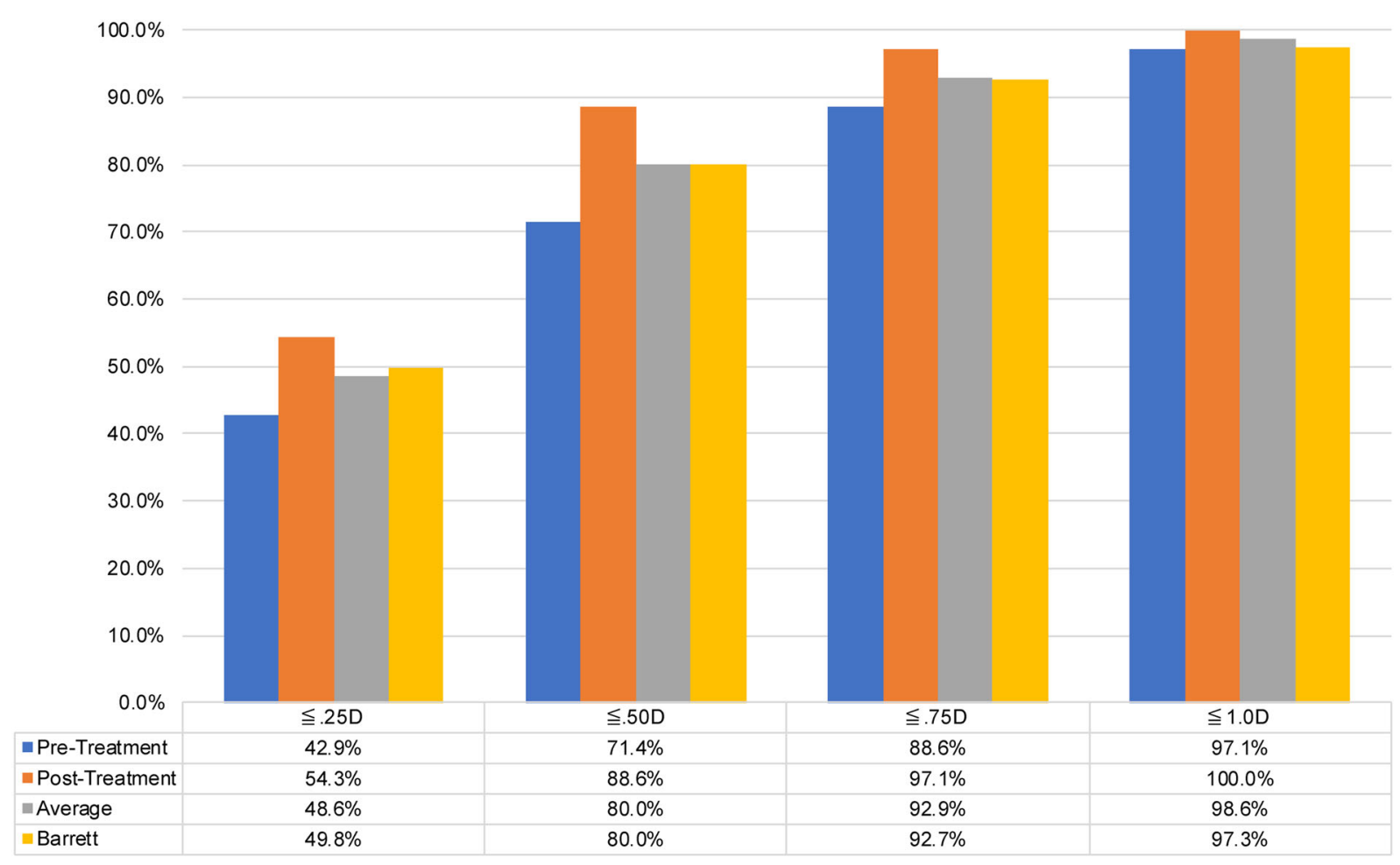

Fig. 1 Accuracy of predicted postoperative spherical equivalent (P-SE). Pre-treatment, pre-dry eye treatment with rebamipide; post-treatment, post-dry eye treatment with rebamipide; Average, average of both pre- and postdry eye treatment with rebamipide; Barrett, reference data from a large-scale study [18]. $X$ axis: absolute difference

category, the accuracy of P-SE after dry eye treatment was significantly higher than that before dry eye treatment $(p<0.01)$.

The average accuracy of P-SE for pre- and post-dry eye treatment was within $0.25 \mathrm{D}$ in $48.6 \%$, within $0.5 \mathrm{D}$ in $79.8 \%$, within $0.75 \mathrm{D}$ in $92.9 \%$, and within $1.0 \mathrm{D}$ in $98.6 \%$ of patients. Figure 1 also shows the comparison between the average accuracy of P-SE for both pre- and postdry eye treatment and the previously reported accuracy of P-SE based on the Barrett Universal II IOL calculation equation [18].

TBUT increased significantly after dry eye treatment $(p<0.01$; Table 1$)$. After dry eye treatment, C-SPK and HOAs decreased significantly $(p<0.01$; Table 1$)$. The most relevant datasets are presented in Table S1 and Table S2. between subjective postoperative spherical equivalent and predicted postoperative spherical equivalent; $D$ diopter. $Y$ axis: proportion of patients

Table 1 Difference in TBUT, HOAs, and C-SPK preand post-dry eye treatment

\begin{tabular}{llll}
\hline & Pre-treatment & Post-treatment & $\boldsymbol{p}$ \\
\hline TBUT & $2.67 \pm 1.02$ & $4.20 \pm 0.98$ & $p<0.01$ \\
HOAs & $0.32 \pm 0.04$ & $0.27 \pm 0.03$ & $p<0.01$ \\
C-SPK & $0.97 \pm 0.89$ & $0.49 \pm 0.56$ & $p<0.01$ \\
\hline
\end{tabular}

TBUT tear breakup time, HOAs higher-order aberrations, $C$-SPK superficial punctate keratopathy in central part of cornea

\section{DISCUSSION}

To the best of our knowledge, no previous study has shown that dry eye treatment with rebamipide ophthalmic suspension improves the refractive accuracy of cataract surgery. Our 
findings demonstrated a significant improvement in the accuracy of P-SE after dry eye treatment with rebamipide.

These results are comparable to those of a recently published study which indicated that dry eye treatment with another eye drop, 5\% lifitegrast ophthalmic solution, increased the accuracy of P-SE [11]. In addition, our research also illustrated that the accuracy of P-SE in both pre- and post-dry eye treatment in our study was comparable to that of P-SE calculated with the Barrett Universal II IOL calculation equation in a previous large-scale study [18]. The accuracy of the P-SE pre-dry eye treatment was lower and that post-dry eye treatment was higher than the values indicated in the previous largescale study [18]. These results indicate the possibility that dry eye treatment with rebamipide may increase the accuracy of well-established IOL calculation equations.

Preoperative dry eye treatment with rebamipide can be a useful option for improving the quality of vision and increasing patient satisfaction after cataract surgery. A previous study demonstrated that patients with dry eye have larger HOAs than patients without dry eye, and the increase in HOAs in dry eyes is mainly induced by an increase in tear film instability [19]. Another study theorized that tear film instability causes the tear film to break up rapidly and thicken irregularly [13]. Roh et al. [20] reported that corneal power measurements determined with the IOLMaster 700 (Carl Zeiss Meditec AG) were less accurate in patients with marked HOAs. Furthermore, Koh et al. reported that the number of HOAs measured in patients with dry eye and C-SPK was higher than those measured in patients with dry eye without C-SPK [21]. Previous studies have indicated the effectiveness of rebamipide in improving HOAs $[21,22]$. It has also been reported that rebamipide positively affects the corneal epithelial layer by improving the structural irregularities and increasing the growth of corneal epithelial cells, which leads to the improvement of C-SPK $[13,23]$. These reports support our findings, where TBUT increased and C-SPK decreased significantly following dry eye treatment with rebamipide. These findings indicate that ocular surface condition improved after dry eye treatment. As a result, HOAs, which quantify corneal irregularity, also decreased significantly.

Additionally, previous studies have demonstrated that the improvement in ocular surface irregularity increased the accuracy of measurement of corneal power and predicted postoperative refraction $[7,11,24]$. Our main findings, where P-SE significantly improved after dry eye treatment with rebamipide, may be supported by these reports. To the best of our knowledge, this has not been reported previously.

There are some limitations to our study. One limitation of this research is the absence of a control group. Preservative-free artificial tears may show a similar effect on variables in this study. This should be investigated in further research. Several previous studies, including our study, used $0.25 \mathrm{D}$ error to determine the accuracy of postoperative refraction; however, since most manufactured IOLs are in steps of 0.5 $\mathrm{D}$, an error of $0.25 \mathrm{D}$ may be inevitable. Therefore, this should be taken into consideration. Additionally, we investigated the data using only the Barrett Universal II IOL calculation formula. The result may be different from that with other IOL calculation formulas, which include keratometry as one of the variables. Therefore, it may be more convincing if the same results are seen with other IOL calculation formulas in future research. Regarding the sample size, post hoc power analysis revealed $80 \%$ power to detect medium $(d=0.5)$ effect sizes by the Wilcoxon signed-rank test, but the sample size was still relatively small. Therefore, we may consider the findings of these tests to be strongly indicative rather than conclusive. Another limitation is that we used the Japanese definition of dry eye, which is different from that of other scientific definitions, such as the Adelphi and DEWS dry eye study [25]. Epidemiological studies showed that the short TBUT type of dry eye is more common in Japan and other Asian countries compared to the other types of dry eye disease [26, 27]. The Asia Dry Eye Society (ADES) was established in 2014, and they formulated the current definition of dry eye and its diagnostic criteria [28]. Regarding the severity of dry eye, ADES reported a discrepancy between severe symptoms and severe signs (ocular surface damage) in the short 
TBUT type of dry eye [28]. However, further research using other dry eye definitions is necessary to evaluate whether these different definitions of dry eye could affect our findings. Additionally, we evaluated C-SPK using a basic four-point scale of the National Eye Institute/ Industry Workshop method. The use of a different scoring system for regional SPK, such as the Oxford grading system [29], may be more suitable for scrutinizing how C-SPK affects corneal surface conditions and the accuracy of P-SE.

Lastly, comparing the effectiveness of different types of dry eye treatments in improving refractive accuracy in cataract surgery may be of value.

\section{CONCLUSION}

This study demonstrated that preoperative dry eye treatment with rebamipide may be useful in improving the quality of ocular surface conditions, as evidenced by the increase in TBUT and reduction in HOAs and C-SPK scores. These effects can lead to an increase in the accuracy of predicting postoperative refraction. Preoperative dry eye treatment with rebamipide may be an option for improving the accuracy of postoperative refraction calculation. The results of this research imply that other dry eye treatment methods may also have the ability to improve the refractive accuracy of cataract surgery and that preoperative dry eye treatment may be a promising option for increasing patient satisfaction.

\section{ACKNOWLEDGEMENTS}

Funding. No funding or sponsorship was received for this study or publication of this article. The journal's Rapid Service Fee was funded by the authors.

Medical Writing, Editorial, and Other Assistance. We would like to thank Editage (www.editage.com) for English language editing.
Authorship. All named authors meet the International Committee of Medical Journal Editors (ICMJE) criteria for authorship for this article, take responsibility for the integrity of the work as a whole, and have given their approval for this version to be published.

Author Contributions. Takeshi Teshigawara, Akira Meguro, and Nobuhisa Mizuki made substantial contributions to the conception and design, data acquisition, or data analysis and interpretation. Takeshi Teshigawara and Akira Meguro drafted the article and critically revised it for important intellectual content.

Disclosures. Takeshi Teshigawara, Akira Meguro and Nobuhisa Mizuki have no competing interests to declare for this research.

Compliance with Ethics Guidelines. The ethical committees of Yokosuka Chuo Eye Clinic and Tsurumi Chuo Eye Clinic approved this study. The study adhered to the tenets of the Declaration of Helsinki throughout the data collection process. After providing detailed information regarding this research, written consent was obtained from all patients for participation in this research and the publication of the resulting data.

Data Availability. Most relevant datasets are included in this published article and supplementary information file. Other datasets are also available from the corresponding author upon reasonable request.

Authors' Information. Takeshi Teshigawara: MD, PhD, MBA; Director of the Yokosuka Chuoh Eye Clinic; Clinical associate professor of the Department of Ophthalmology of Yokohama City University School of Medicine. Akira Meguro: PhD; Specially appointed Associate Professor of the Department of Ophthalmology, Yokohama City University School of Medicine. Nobuhisa Mizuki: MD, PhD; Professor of the Department of Ophthalmology, Yokohama City University School of Medicine.

Open Access. This article is licensed under a Creative Commons Attribution- 
NonCommercial 4.0 International License, which permits any non-commercial use, sharing, adaptation, distribution and reproduction in any medium or format, as long as you give appropriate credit to the original author(s) and the source, provide a link to the Creative Commons licence, and indicate if changes were made. The images or other third party material in this article are included in the article's Creative Commons licence, unless indicated otherwise in a credit line to the material. If material is not included in the article's Creative Commons licence and your intended use is not permitted by statutory regulation or exceeds the permitted use, you will need to obtain permission directly from the copyright holder. To view a copy of this licence, visit http:// creativecommons.org/licenses/by-nc/4.0/.

\section{REFERENCES}

1. Miyata K, Yoshikawa T, Mine M, et al. Cataract surgery and visual acuity in elderly Japanese: results of Fujiwara-kyo eye study. Biores Open Access. 2017;6:28-34. https://doi.org/10.1089/biores.2017. 0007 .

2. Mollazadegan K, Lundström M. A study of the correlation between patient-reported outcomes and clinical outcomes after cataract surgery in ophthalmic clinics. Acta Ophthalmol. 2015;93:293-8. https://doi.org/10.1111/aos.12490.

3. Gibbons A, Ali TK, Waren DP, Donaldson KE. Causes and correction of dissatisfaction after implantation of presbyopia-correcting intraocular lenses. Clin Ophthalmol. 2016;10:1965-70. https:// doi.org/10.2147/OPTH.S114890.

4. Courville CB, Smolek MK, Klyce SD. Contribution of the ocular surface to visual optics. Exp Eye Res. 2004;78:417-25. https://doi.org/10.1016/j.exer. 2003.10.012.

5. Dong J, Zhang Y, Zhang H, Jia Z, Zhang S, Wang X. Comparison of axial length, anterior chamber depth and intraocular lens power between IOLMaster and ultrasound in normal, long and short eyes. PLoS ONE. 2018;13: e0194273. https://doi. org/10.1371/journal.pone.0194273.

6. Koh S. Irregular astigmatism and higher-order aberrations in eyes with dry eye disease. Invest
Ophthalmol Vis Sci. 2018;59:DES36-40. https://doi. org/10.1167/iovs.17-23500.

7. Teshigawara T, Meguro A, Mizuki N. Effects of rebamipide on differences in power and axis of corneal astigmatism between two intra-patient keratometric measurements in dry eyes. Ophthalmol Ther. 2021;10:891-904. https://doi.org/10. 1007/s40123-021-00368-9.

8. Epitropoulos AT, Matossian C, Berdy GJ, Malhotra RP, Potvin R. Effect of tear osmolarity on repeatability of keratometry for cataract surgery planning. J Cataract Refract Surg. 2015;41:1672-7. https://doi. org/10.1016/j.jcrs.2015.01.016.

9. Trattler WB, Majmudar PA, Donnenfeld ED, McDonald MB, Stonecipher KG, Goldberg DF. The prospective health assessment of cataract patients' ocular surface (PHACO) study: the effect of dry eye. Clin Ophthalmol. 2017;11:1423-30. https://doi. org/10.2147/OPTH.S120159.

10. Koh S, Maeda N, Ogawa M, et al. Fourier analysis of corneal irregular astigmatism due to the anterior corneal surface in dry eye. Eye Contact Lens. 2019;45:188-94. https://doi.org/10.1097/ICL. 0000000000000559 .

11. Hovanesian J, Epitropoulos A, Donnenfeld ED, Holladay JT. The effect of Lifitegrast on refractive accuracy and symptoms in dry eye patients undergoing cataract surgery. Clin Ophthalmol. 2020;14: 2709-16. https://doi.org/10.2147/OPTH.S264520.

12. Koh S, Maeda N, Hori Y, et al. Effects of suppression of blinking on quality of vision in borderline cases of evaporative dry eye. Cornea. 2008;27:275-8. https://doi.org/10.1097/ICO.0b013e31815be9c8.

13. Urashima H, Okamoto $\mathrm{T}$, Takeji $\mathrm{Y}$, Shinohara $\mathrm{H}$, Fujisawa S. Rebamipide increases the amount of mucin-like substances on the conjunctiva and cornea in the $\mathrm{N}$-acetylcysteine-treated in vivo model. Cornea. 2004;23:613-9. https://doi.org/10.1097/01. ico.0000126436.25751.fb.

14. Shimazaki J. Concept of new definition and diagnostic criteria of dry eye disease in 2016. Atarashii Ganka. 2018;35:853-7 (in Japanese).

15. Midorikawa-Inomata A, Inomata $T$, Nojiri $S$, et al. Reliability and validity of the Japanese version of the ocular surface disease index for dry eye disease. BMJ Open. 2019;9: e033940. https://doi.org/10. 1136/bmjopen-2019-033940.

16. Lemp MA. Report of the National Eye Institute/ Industry workshop on clinical trials in dry eyes. CLAO J. 1995;21:221-32. 
17. Barrett GD. An improved universal theoretical formula for intraocular lens power prediction. J Cataract Refract Surg. 1993;19:713-20. https://doi. org/10.1016/s0886-3350(13)80339-2.

18. Melles RB, Holladay JT, Chang WJ. Accuracy of intraocular lens calculation formulas. Ophthalmology. 2018;125:169-78. https://doi.org/10.1016/ j.ophtha.2017.08.027.

19. Koh S, Maeda N, Hirohara Y, et al. Serial measurements of higher-order aberrations after blinking in patients with dry eye. Invest Ophthalmol Vis Sci. 2008;49: 133-8. https://doi.org/10.1167/iovs.07-0762.

20. Roh HC, Chuck RS, Lee JK, Park CY. The effect of corneal irregularity on astigmatism measurement by automated versus ray tracing keratometry. Medicine (Baltim). 2015;94: e677. https://doi.org/ 10.1097/MD.0000000000000677.

21. Koh S, Inoue Y, Sugmimoto T, Maeda N, Nishida K. Effect of rebamipide ophthalmic suspension on optical quality in the short break-up time type of dry eye. Cornea. 2013;32:1219-23. https://doi.org/ 10.1097/ICO.0b013e318294f97e.

22. Inoue $\mathrm{Y}$, Ochi S, Koh S. Effect of $2 \%$ rebamipide ophthalmic suspension on perioperative period of cataract surgery in dry eye patients. Atarasii Ganka (J Eye). 2016;33:1363-8.

23. Tanito M, Takanashi T, Kaidzu S, Yoshida Y, Ohira A. Cytoprotective effects of rebamipide and carteolol hydrochloride against ultraviolet B-induced corneal damage in mice. Invest Ophthalmol Vis Sci. 2003;44:2980-5. https://doi.org/10.1167/iovs.021043.
24. Matossian C. Impact of thermal pulsation treatment on astigmatism management and outcomes in Meibomian gland dysfunction patients undergoing cataract surgery. Clin Ophthalmol. 2020;14: 2283-9. https://doi.org/10.2147/OPTH.S263046.

25. Miyata K, Amano S, Sawa M, Nishida T. A novel grading method for superficial punctate keratopathy magnitude and its correlation with corneal epithelial permeability. Arch Ophthalmol. 2003;121:1537-9. https://doi.org/10.1001/ archopht.121.11.1537.

26. Kawashima M, Yamada M, Suwaki K, et al. A clinicbased survey of clinical characteristics and practice pattern of dry eye in Japan. Adv Ther. 2017;34: 732-43. https://doi.org/10.1007/s12325-017-0487$\mathrm{x}$.

27. Uchino M, Yokoi N, Uchino Y, et al. Prevalence of dry eye disease and its risk factors in visual display terminal users: the Osaka study. Am J Ophthalmol. 2013;156:759-66. https://doi.org/10.1016/j.ajo. 2013.05.040.

28. Tsubota K, Yokoi N, Shimazaki J, et al. New perspectives on dry eye definition and diagnosis: a consensus report by the Asia Dry Eye Society. Ocul Surf. 2017;15:65-76. https://doi.org/10.1016/j.jtos. 2016.09.003.

29. Bron AJ, Evans VE, Smith JA. Grading of corneal and conjunctival staining in the context of other dry eye tests. Cornea. 2003;22:640-50. https://doi. org/10.1097/00003226-200310000-00008. 